\title{
Erratum in: Budget Impact Analysis of anakinra in the treatment of patients with Still's Disease
}

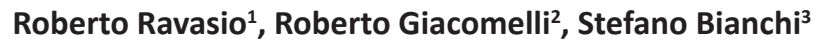 \\ ${ }^{1}$ Health Publishing \& Services S.r.l., Milan - Italy \\ ${ }^{2}$ Department of Biotechnological and Applied Clinical Science, Rheumatology Unit, School of Medicine, University of L'Aquila, L'Aquila - Italy \\ ${ }^{3}$ Unità Operativa Servizio farmaceutico ospedaliero e territoriale Azienda UsI Ferrara - Italy
}

In the article, "Budget Impact Analysis of anakinra in the treatment of patients with Still's Disease", ${ }^{[1]}$ which appeared in Volume 7, Issue 1 of Global \& Regional Health Technology Assessment, the number of patients undergoing treatment with tocilizumab and anakinra displayed in Table $\mathrm{V}$ (column Current Scenario) was inadvertently inverted. Affected data have been corrected in the article now appearing online. The authors apologize for any inconvenience caused to the readers by these changes, which do not affect the final results of the study.
The final version of this article is available online and includes a reference to this correction.

\section{Reference}

1. Ravasio R. Giacomelli R, Bianchi S, Budget Impact Analysis of anakinra in the treatment of patients with Still's Disease Glob Reg Health Technol Assess 2020; 7(1): 72-80. https://doi.org/ 10.33393/grhta.2020.2140 